\title{
The fern sporangium: a unique catapult
}

2

3

4

5

6

7

8

9

10

11

12

13

14

15

16

17

18

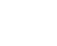

\author{
X. Noblin ${ }^{1, *}$, N. Rojas ${ }^{2}$, J. Westbrook ${ }^{3}$, C. LLorens ${ }^{2}$, M. Argentina ${ }^{2} \&$ J. \\ Dumais $^{4}$
}

6

1 LPMC, UMR 6622 CNRS-UNS, Parc Valrose, 06108 Nice Cedex 2, France.

2 LJAD, UMR 6621 CNRS-UNS, Parc Valrose, 06108 Nice Cedex 2, France.

3 Department of Botany, University of Florida, Gainesville, FL, 32611 USA.

4 Organismic and Evolutionary Biology, Harvard University, Cambridge, MA, 02138

$$
\text { USA. }
$$

(1)

2

Spore dispersal in plants and fungi plays a critical role in the survival of species

and is thus under strong selective pressure. As a result, various plant and fungal groups

have evolved ingenious mechanisms to disperse their spores effectively $[1,2]$. Many of

these mechanisms use the same physical principles as man-made devices but often

achieve better performance. One such dispersal mechanism is the cavitation-triggered

catapult of fern sporangia. The sporangia open when dehydrating and use the stored elastic energy to power a fast closure motion that ultimately ejects the spores. The beauty of this dispersal mechanism and similarity with medieval catapults has not escaped notice [1]. All man-made catapults are equipped with a crossbar to stop the motion of the arm midway. Without it, catapults would launch their projectiles right into the ground. This "crossbar" is conspicuously missing from the sporangium, suggesting that it should simply speed up to its closed conformation without ejecting the 
24 spores. Here we show that much of the sophistication of this ejection mechanism, and 25 the basis for its efficiency, lie in the two very different time scales associated with the 26 sporangium closure. The simple structure of the sporangium belies the complexity of its 27 action (Fig. 1A). Central to the ejection process is the annulus - a row of 12-13 cells that forms a crest to one side of a spherical capsule enclosing the spores. As the annulus cells lose water by evaporation, their thickened radial walls are forced closer together 30 while lateral walls collapse internally (Fig. 1B, movie S1). The whole annulus is thus 31 bent out of shape much like an accordion in the hands of a musician. The strong change 32 in curvature (Fig. 1B\&D) forces the opening of the sporangium at the stomium, thus 33 exposing the spores. All the while, water tension builds in the cells of the annulus [3, 4]. 34 When the tension reaches a critical value (approximately $-9 \mathrm{MPa}$ [5]), cavitation occurs 35 within adjacent cells [6] (Fig. 1C\&S2, movie S2\&S3). The annulus then closes by about $3630-40 \%$ within about $10 \mu \mathrm{s}$, leading to a quick release of the energy stored in the 37 annulus and expulsion of the spores at an initial velocity of up to $10 \mathrm{~m} \cdot \mathrm{s}^{-1}$ [7]. This 38 corresponds to an acceleration of approximately $10^{5} \mathrm{~g}$. This first phase is followed by a comparatively slow relaxation to a $85 \%$ closed configuration in a few hundreds of ms.

40 We interpret the two time scales as a fast inertial recoil of the annulus followed by a 41 slow poroelastic dissipation [8] of the energy remaining in the annulus. The annulus 42 walls are constituted of a tight network of cellulose fibers surrounded by water that 43 flows to conform to their relative displacements. The tiny size of the pores [9] and thick 44 wall [10] induce strong viscous losses (from Darcy's law) that dramatically slow down 45 the annulus motion. This dynamics can be described using a generalized viscoelastic 46 Maxwell model that fits our data very well and integrates all the physical forces at play 47 (see Fig. 1E\&S3). The measured and predicted time scales are in good agreement both 
48 for the inertial (respectively $25 \mu s$ and $27 \mu s$ ) and the poroelastic regime (respectively

$495.8 \mathrm{~ms}$ and $3 \mathrm{~ms}$ ). The coexistence of these two widely different timescales allows the

50 sporangium to release its spores efficiently without the use of structural elements to 51 arrest the recoil motion.

52 It is striking that a dozen cells placed in a row can fulfill all the functions of a 53 medieval catapult including the motive force for charging the catapult (water cohesion), 54 energy storage (annulus wall), triggering mechanism (cavitation), and returning motion 55 arrest (poroelastic behavior of the annulus wall).

\section{References and Notes}

58 1. C. T. Ingold, Spore Liberation (Clarendon Press, Oxford, 1965).

59 2. X. Noblin et al., J. Exp. Biol. 212, 2835 (2009).

60 3. O. Renner, Jahr. wiss. Bot. 56, 647 (1915).

61 4. A. Ursprung, Ber. deut. bot. Ges 33, 153 (1915).

62 5. X. Noblin et al., Proc. of the 6th Plant Biomechanics Conference, Cayenne, French $63 \quad$ Guyana (2009)

64 6. K. T. Ritman, J. A. Milburn, J. Exp. Bot. 41, 1157 (1990).

65 7. A. L. King, Proc. Nat. Acad. Sci. 30, 155 (1944).

66 8. J. M. Skotheim, L. Mahadevan, Proc. Roy. Soc. Lond. A 460, 1995 (2004).

67 9. N. Carpita et al. SCIENCE 205, 4411, pp. 1144-1147 (1979)

68 10. K. Haider. Planta 44, pp. 370-411 (1954)

69

\section{Acknowledgments}



project: "EVAPLANTE"), the ANR (project "CAVISOFT") and the CONICYT.

73

\section{Supporting Online Material}

75

76

77

78

79

80

81

82 Caption for Fig.1: (A) The sporangium of Polypodium aureum. Annulus geometry

83 during sporangium opening in (B) and in (C) just prior to cavitation, $0.4 \mathrm{~ms}$ and $40 \mathrm{~ms}$

84 after cavitation. Note the seven cells that have cavitated (red arrows). (D) Annulus

85 curvature during the opening (blue) and closing (red) phases. (E) The closing phase in

86 log-linear scale revealing the poroelastic relaxation, the green line represents the fit from

87 our model. The numbers correspond to frames in $(\mathbf{B})$ and $(\mathbf{C})$. Insert: expended view of 88 the inertial oscillations. 


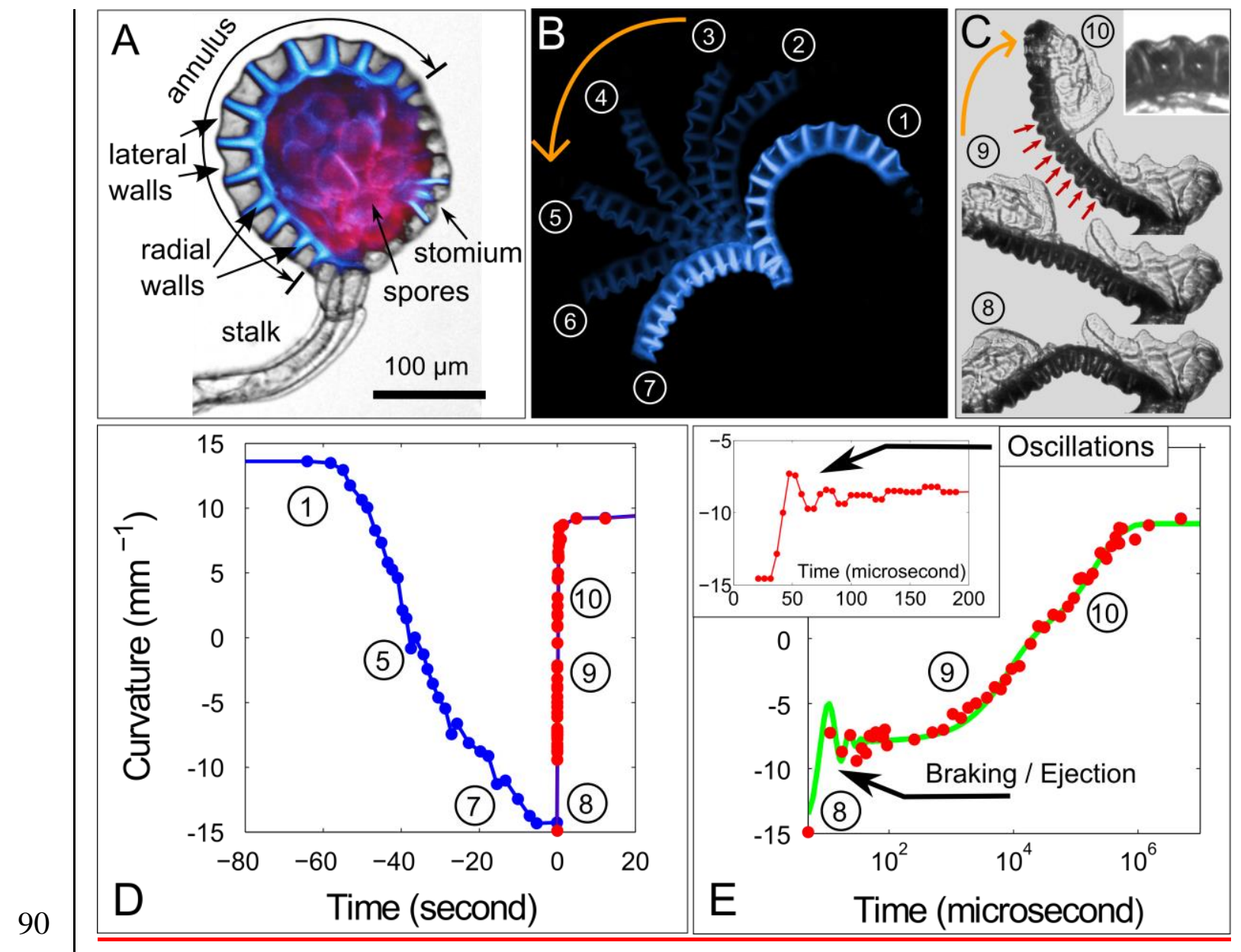

